the Lyman- $\alpha$ emission line, which is Doppler broadened by speeds up to about $1,000 \mathrm{~km} \mathrm{~s}^{-1}$. A spectrum with the slit aligned along the long axis of the cloud gives some indication of a systematic velocity gradient of about $\pm 500 \mathrm{~km} \mathrm{~s}^{-1}$. There are also weak emission lines from ionized carbon, radiated at wave-lengths of 1,909 $\AA\left(\mathrm{C}_{\mathrm{III}}\right)$ and $2,325 \AA\left(\mathrm{C}_{\mathrm{II}}\right)$, with the same redshift as the Lyman- $\alpha$ and similarly distributed over the face of the cloud.

Hence the cloud is not a primordial object because it already contains heavy elements. D. Kunth and I, however, in a discussion on the absence of nearby primordial galaxies (Astrophys. J. 300, $496-499 ; 1987$ ) showed that the hot stars which ionize the gas also produce and eject heavy elements on a timescale of only a few million years. Therefore, it is quite possible that the unionized (invisible) parts of the cloud are pure hydrogen and helium formed in the Big Bang.

The source of the ionizing radiation in the cloud is indeed likely to be young, hot, massive stars, as there is no indication either in the optical or the radio data of quasar-like activity that could provide an alternative source of ionizing photons. As McCarthy et al. point out, the origin of the strong radio emission is mysterious. It is possible, however, that the burst of star formation which is inferred may have been triggered by the motion of the radio blobs through the gas; similar phenomena have been observed recently in nearby radio galaxies. The inferred rate of star formation is about 70 solar masses $\left(M_{\odot}\right)$ per year in the form of stars more massive than $10 M_{\odot}$ and about $500 M_{\odot}$ per year if a correction is made for the mass in stars that produce negligible ionizing radiation.

McCarthy et al. estimate that the mass of ionized hydrogen in the cloud is less than $10^{11} M_{\odot}$ on the assumption that the gas is uniformly distributed throughout its volume. This upper limit could be reduced by orders of magnitude if the gas is clumped. Thus, if it is distributed in dense regions of ionized hydrogen around hot stars, only about $10^{7} M_{\odot}$ would be required. The authors remark that the total mass in stars and gas is unknown. The line width and possible velocity gradient, however, imply a very large total mass, about $2.8 \times 10^{12} M_{\odot}$, if the cloud is gravitationally bound; they do not address this question. The cloud of ionized gas discovered by McCarthy et al. is the nearest approach to a primordial galaxy yet discovered. As they point out, if the cloud really is a proto-galaxy it has made a powerful non-thermal radio source before forming most of its stars. Also, the object is not shrouded in dust as some authors suggest primordial galaxies might be.

Wallace L.W. Sargent is at the Palomar Observatory, California Institute of Technology, Pasadena, California 91125, USA.
AIDS

\section{Receptor molecule blocks HIV}

\author{
Robin A. Weiss
}

SINCE the CD4 (T4) antigen was identified $^{1,2}$ as an essential component of the cell surface receptor for the human immunodeficiency virus (HIV-1), it has become one of the most intensively studied receptors of any enveloped virus. Now five papers, one in Science ${ }^{3}$, and four in this issue of Nature $^{4-7}$ show that soluble forms of CD4 antigen (sCD4) can block the infectivity of the virus. Clinical trials of sCD4 as an antiviral agent are already being planned in the United States.

CD 4 antigen acts as the HIV-1 receptor through the recognition by the outer viralenvelope antigen, gp120, of a site on the receptor molecule ${ }^{8}$ possessing an affinity constant $^{9}$ of about $10^{-9} \mathrm{M}$. Transfer and expression of the CD4 gene to human cells such as HeLa confers susceptibility to HIV-1 infection ${ }^{10}$. (Curiously, this is not the case for mouse cells; although HIV-1 specifically binds to mouse cells expressing human CD4, it does not infect them.) Monoclonal antibodies against CD4 block the binding of HIV-1 and subsequent infection and syncytium induction ${ }^{1,2,8,11}$. Thus, the CD4 antigen presents the initial binding site for HIV-1 to enter the host cell.

The reports of $\mathrm{sCD} 4$ indicate that saturating amounts are needed to block HIV-1 infectivity. In most of the studies complete inhibition of low-titre HIV-1 $(100-1,000$ tissue-culture infectious doses, TCID) was achieved only with microgram amounts of sCD4. Although the Genentech group ${ }^{3}$ shows very high affinity of its SCD4 with gp120, it was barely able to achieve complete blocking of HIV-1 infectivity (100 TCID) with undiluted SCD4 preparations. In contrast, the Smith Kline \& French group ${ }^{6}$ observes greater than 3,000 -fold inhibition of HIV-1 infectivity with $8.6 \mu \mathrm{g} \mathrm{m}^{-1} \mathrm{sCD} 4$. The inhibitory effect of the other sCD4 molecules is intermediate, whether produced from insect $^{5}$ or mammalian ${ }^{6.7}$ cells. This could well reflect variation in the HIV-1 infectivity assays rather than the efficacy of different SCD4 preparations.

The precise binding site on CD4 for viral gp120 is now being analysed in several laboratories by site-specific mutagenesis. Several lines of study indicate that it is near to the epitope recognized by leu $3 \mathrm{a}$ and related monoclonal antibodies. These strongly inhibit HIV-1 binding, infection and syncytium induction $^{11}$, and some anti-idiotypic sera to leu $3 a$ weakly react in virus neutralization assays ${ }^{12-14}$. Moreover, our recent data indicate $^{15}$ that the epitope recognized by leu $3 \mathrm{a}$ is highly conserved in primate evolution, and that cell culture infection by HIV-1 of New World monkeys such as marmosets is blocked by the monoclonal antibody leu $3 \mathrm{a}$. One would therefore surmise that HIV-1 binds to a CD4 epitope necessarily conserved for the normal physiological role this antigen plays in regulation of the immune system, thought to be interaction with class II antigens of the major histocompatiblity complex $^{16}$. If so, one might expect sCD4 itself to be immunosuppressive. However, Reinherz's group shows ${ }^{5}$ that its sCD4 does not inhibit class II-dependent immune killing by a $\mathrm{CD}^{+}$cytolytic cell clone. This result provides encouragement for clinical trials of sCD4, which may serve in vivo not only to block spreading HIV-1 infection, but also to sequester free viral gp120 and reduce its possible immune toxicity in AIDS (acquired immune deficiency syndrome).

It remains to be determined how small a fragment of CD4 is able to inhibit HIV-1 infectivity. Most of the sCD4 preparations reported $^{3-7}$ comprise almost all of the extracellular domains of the antigen, although Traunecker et al. show that a fragment containing only the two aminoterminal immunoglobulin-like domains is almost as inhibitory as the whole extracellular portion. All the groups ${ }^{3-7}$ appear to have used a single strain of HIV-1 in the sCD4 inhibition tests, but our own unpublished observations indicate that $\mathrm{SCD} 4$ inhibits all strains of HIV-1, HIV-2 and related simian immunodeficiency viruses tested. This is not surprising as all these viruses appear to recognize the same domain of CD4 as their binding receptor ${ }^{14}$.

HIV entry to non-lymphoid cells might be mediated by receptors other than CD4, including the infection of monocytes via $\mathrm{Fc}$ receptors. It is important to determine whether SCD4 inhibition of HIV infection applies solely to CD4-mediated entry.

\footnotetext{
1. Dalgleish, A.G. et al. Nature. 312, 736-766 (1984)

2. Klatzmann, D. et al. Nature 312, 767-768 (1984).

3. Smith, D.H. et al. Science 238, 1704-1707 (1987)

4. Fisher, R.A. et al. Nature 331, 76-78 (1988).

5. Hussey, R.E. et al. Nature 331, 78-81 (1988).

6. Deen, K.C. et al. Nature 331, 82-84 (1988).

7. Traunecker, A., Lüke, W. \& Karjalainen, K. Nature 331 84-86 (1988).

8. McDougal, J. et al. Science 231, 382-385 (1986).

9. Lasky, L. et al. Cell 50, 975-985 (1987).

10. Maddon, P.J. et al. Cell 42, 685-698 (1986).

11. Sattenau, Q.J., Dalgleish, A.G., Weiss, R.A. \& Beverley, P.C.L. Science 234, 1120-1123 (1986).

12. Chanh, T. et al. Proc, natn. Acad. Sci. U.S.A. 84, 3891 3895 (1987).

13. Dalgleish, A.G. et al. Lancet ii, 1047-1050 (1987).

14. Sattentau, Q.J. et al. Int. Conf. AIDS III 160 (1987).

15. McClure, M.O. et al. Nature 330, 487-489 (1987).

16. Doyle, C. \& Strominger, J.L. Nature 330, 256-259 (1987).
}

Robin A. Weiss is at the Institute of Cancer Research, Chester Beatty Laboratories, Fulham Road, London SW3 6JB, UK. 